\title{
No effect of low-level chronic neonicotinoid exposure on bumblebee learning and fecundity
}

Saija Piiroinen, Cristina Botías, Elizabeth Nicholls, Dave Goulson

In recent years, many pollinators have declined in abundance and diversity worldwide, presenting a potential threat to agricultural productivity, biodiversity and functioning of natural ecosystems. One of the most debated factor proposed to be contributing to pollinator declines is exposure to pesticides, particularly neonicotinoids, a widely used class of systemic insecticide. Also, newly emerging parasites and diseases, thought to be spread via contact with managed honeybees, may pose threats to other pollinators such as bumblebees. Compared to honeybees, bumblebees could be particularly vulnerable to the effects of stressors due to their smaller and more short-lived colonies. Here, we studied the effect of field-realistic, chronic clothianidin exposure and inoculation with the parasite Nosema ceranae on survival, fecundity, sugar water collection and learning using queenless Bombus terrestris audax microcolonies in the laboratory. Chronic exposure to 1 ppb clothianidin had no significant effects on the traits studied. Interestingly, pesticide exposure in combination with additional stress caused by harnessing bees for proboscis extension response (PER) learning assays, led to an increase in mortality. In contrast to previous findings, the bees did not become infected by $N$. ceranae after experimental inoculation with the parasite spores, suggesting variability in host resistance or parasite virulence. However, this treatment induced a slight, short term reduction in sugar water collection, potentially through stimulation of the immune system of the bees. Our results suggest that chronic exposure to 1 ppb clothianidin does not have adverse effects on bumblebee fecundity or learning ability. 
1 No effect of low-level chronic neonicotinoid exposure on bumblebee

2 learning and fecundity

3 Saija Piiroinen, Cristina Botías, Elizabeth Nicholls \& Dave Goulson

4

5 School of Life Sciences, University of Sussex, Brighton, BN1 9QG, UK

6

7 Corresponding author:

8 Saija Piiroinen

9 School of Life Sciences, University of Sussex, Brighton, BN1 9QG, UK

10 Email: saija.p.piiroinen@gmail.com

11 


\section{Abstract}

13 In recent years, many pollinators have declined in abundance and diversity worldwide,

14 presenting a potential threat to agricultural productivity, biodiversity and functioning of natural

15 ecosystems. One of the most debated factor proposed to be contributing to pollinator declines is

16 exposure to pesticides, particularly neonicotinoids, a widely used class of systemic insecticide.

17 Also, newly emerging parasites and diseases, thought to be spread via contact with managed

18 honeybees, may pose threats to other pollinators such as bumblebees. Compared to honeybees,

19 bumblebees could be particularly vulnerable to the effects of stressors due to their smaller and

20 more short-lived colonies. Here, we studied the effect of field-realistic, chronic clothianidin

21 exposure and inoculation with the parasite Nosema ceranae on survival, fecundity, sugar water

22 collection and learning using queenless Bombus terrestris audax microcolonies in the laboratory.

23 Chronic exposure to $1 \mathrm{ppb}$ clothianidin had no significant effects on the traits studied.

24 Interestingly, pesticide exposure in combination with additional stress caused by harnessing bees

25 for proboscis extension response (PER) learning assays, led to an increase in mortality. In

26 contrast to previous findings, the bees did not become infected by $N$. ceranae after experimental

27 inoculation with the parasite spores, suggesting variability in host resistance or parasite

28 virulence. However, this treatment induced a slight, short term reduction in sugar water

29 collection, potentially through stimulation of the immune system of the bees. Our results suggest

30 that chronic exposure to $1 \mathrm{ppb}$ clothianidin does not have adverse effects on bumblebee

31 fecundity or learning ability. 
33

34

35

36

37

38

\section{Introduction}

In recent decades many pollinators have declined in abundance or contracted their ranges, presenting a potential threat to agricultural productivity and biodiversity (Biesmeijer et al., 2006; Potts et al., 2010). Insect pollinators are crucial for agricultural productivity providing pollination services to a wide variety of crops with an estimated global value of $€ 153$ billion per year (Gallai et al., 2009; Potts et al., 2010). Moreover, pollinators have a key role in maintaining wild plant communities with over two thirds of flowering plants being dependent on pollinators to reproduce (Ollerton, Winfree \& Tarrant, 2011). Thus there is an urgent need to understand the underlying causes driving current pollinator declines.

One of the most debated factor proposed to be contributing to pollinator declines is exposure to pesticides, particularly neonicotinoids. Widely used as seed dressings and foliar sprays for arable and horticultural crops (Jeschke et al., 2011), the systemic nature of neonicotinoids means that low concentrations of these pesticides may be present in the nectar and pollen of treated crops (European Food Safety Authority, 2012; Sanchez-Bayo \& Goka, 2014; Bonmatin et al., 2015). Furthermore, residual levels of neonicotinoids can also be detected in wild flowers growing near treated crops (Krupke et al., 2012; Botías et al., 2015), meaning many flower-visiting insects may be exposed to sub-lethal doses of neonicotinoids in agricultural landscapes. However, there is a lack of consensus on whether typical levels of exposure have significant impacts on pollinators (Goulson, 2013). Some studies have reported no significant lethal (Cresswell, 2011) or sub-lethal effects of the most commonly used neonicotinoids, imidacloprid, clothianidin or thiamethoxam on honeybees (Schmuck et al., 2001; Pilling et al., 2013; Cutler et al., 2014) or bumblebees (Tasei et al., 2001; Morandin \& Winston 2003; Franklin, Winston \& Morandin, 2004; Laycock et al., 2014) while others have indicated that exposure to sub-lethal levels of the same pesticides can cause significant harm and disruption of behaviour (Tasei, Lerin \& Ripault, 2000; Whitehorn et al., 2012; Gill, Ramos-Rodriguez \& Raine, 2012; Laycock \& Cresswell, 2013; Moffat et al., 2015; Rundlöf et al., 2015). Acting as nicotinic acetylcholine receptor (nAChRs) agonists, very low levels of neonicotinoids can disrupt neuronal functioning in bees (Palmer et al., 2013; Moffat et al., 2015), including parts of the brain essential in learning and memory (Zars, 2000). Impaired learning ability may be one potential mechanism underlying observed reductions in the homing ability and foraging 
63 behaviour of pesticide exposed bees (Schneider et al., 2012; Henry et al., 2012; Gill, Ramos-

64 Rodriguez \& Raine, 2012; Feltham, Park \& Goulson, 2014; Gill \& Raine, 2014).

Another potential factor driving pollinator declines is exposure to newly emerging diseases

66 (Meeus et al., 2011; Ravoet et al., 2013). Anthropogenic movement of honeybee and bumblebee

67 colonies has inadvertently spread bee parasites and pathogens beyond their native range

68 (Plischuk et al., 2009; Graystock, Goulson \& Hughes, 2014). For instance, European honeybees

69 (Apis mellifera) are now widely infected with the microsporidian gut parasite Nosema ceranae,

70 transmitted via ingestion of spores that are spread in faeces or via food exchange (Smith, 2012), of which the putatively original host is the Asian honeybee (A. cerana) (Gómez-Moracho et al., 2015). N. ceranae appears to be highly infective in European honeybees and has been shown to affect honeybee behaviour and survival (Higes et al., 2007; Naug \& Gibbs, 2009). More recently, $N$. ceranae has been reported to infect also bumblebees in Europe and South-America (Plischuk et al., 2009; Graystock et al., 2013; Fürst et al. 2014). However, very little is known about the virulence or possible effects the parasite may pose on bumblebees (but see Graystock et al., 2013, Fürst et al. 2014).

There is a major gap in knowledge concerning the effects of field-realistic exposure to neonicotinoids on other pollinator taxa, such as bumblebees. Bumblebees play a major role in providing pollinating services to wild and crop plants, and their smaller and more short-lived colonies could make them more susceptible to the effects of stressors (Goulson, 2010; Cresswell et al., 2012; Fauser-Misslin et al., 2014; Rundlöf et al., 2015). Here, we used bumblebee (Bombus terrestris) microcolonies to investigate the influence of field-realistic (1 ppb) chronic exposure to clothianidin, currently the most widely used neonicotinoid in Europe, on bumblebee survival, fecundity and feeding behaviour. Given the uncertainty whether field-realistic concentrations of neonicotinoids can have impact on bumblebee populations, and the fact that majority of studies showing detrimental effects of neonicotinoid exposure on bees have used concentrations reflecting worst-case scenarios, at the upper range of field-realistic levels, typically 10 ppb or above (e.g. Henry et al., 2012; Fischer et al., 2014; Scholer \& Krischik, 2014), we chose to use a conservative level within the field-realistic range to better mimic the scenario the bees are most likely to experience in the field conditions. Reported values of the 
94 Bonmatin et al., 2015; Botías et al., 2015). Thus, 1 ppb represents a field-relevant, conservative

95 level. We also inoculated bumblebees with $N$. ceranae to test infectivity and potential harmful

96 effects a challenge with the parasite may pose as to aforementioned traits. Finally, both these

97 stressors have been shown to influence brain functioning and behavioural traits in honeybees and

98 bumblebees (Decourtye et al., 2004; Gegear et al., 2006; Kralj et al., 2007; Aliouane et al., 2009;

99 Moffat et al., 2015; Williamson and Wright, 2013; Yang et al., 2012). Therefore, we investigated

100 whether these factors influence learning ability and memory of individual bees using proboscis

101 extension response (PER) conditioning.

102

103 Materials and methods

104 Bees and pathogen screening

105 Fifteen nests of $B$. terrestris audax with approximately 60 workers were obtained from Biobest

106 (Belgium) via Agralan Ltd (Swindon, UK). They were first screened microscopically and via

107 PCR for common bumblebee and honeybee parasites (Nosema bombi, N. ceranae, Crithidia

108 bombi, Apicystis bombi) by analysing a subset of workers (10\% of workers) immediately upon

109 arrival. A small sample of hind gut, mid gut and malpighian tubules was dissected out and

110 homogenized in $\mathrm{ddH}_{2} \mathrm{O}$. DNA was extracted with $10 \%$ Chelex (Bio-Rad, Hemel Hempstead,

111 Hertfordshire, UK). PCR protocols and parasite-specific primers followed Graystock et al.,

112 (2013).

113

114 Microcolonies and treatments

115 These 15 queenright nests were divided into 60 queenless microcolonies each consisting of 10

116 workers (4 microcolonies per queenright nest). The four microcolonies created from each

117 queenright nest were randomly assigned to one of the four treatments: control, pesticide

118 (clothianidin), parasite (N. ceranae) and exposure to both pesticide and parasite, thus we had a

119 fully factorial design. Using microcolonies instead of whole nests enabled us to control for

120 genetic background. Furthermore, the use of microcolonies is one of the methods recommended

121 by EFSA for risk assessment studies (European Food Safety Authority, 2013). A small amount

122 of brood and wax from the original queenright nest was given to each microcolony to stimulate

123 nest building. Typically in a queenless microcolony, some workers will begin laying unfertilised

124 eggs which develop into male bees. Bees resided in circular plastic containers (diameter $11 \mathrm{~cm}$, 
125 height $9 \mathrm{~cm}$ ) with an aluminium mesh cover to allow air ventilation, and were maintained in the 126 dark at $26^{\circ} \mathrm{C}\left( \pm 1^{\circ} \mathrm{C}\right)$ and $55 \% \mathrm{RH}( \pm 5 \%)$.

127 Microcolonies assigned as pesticide or pesticide+parasite treatment were provided with an 128 ad-lib supply of $60 \%$ sugar water solution contaminated with $1 \mathrm{ppb}$ clothianidin (Sigma-Aldrich, 129 Gillingham, UK) and microcolonies assigned as control or parasite treatments were given 130 untreated food. Stock solutions were dissolved in acetone and dietary concentrations were made 131 on the day of provisioning. Fresh sugar water solutions (contaminated with clothianidin or not) 132 were renewed every third day, and the amount of sugar water solution collected was recorded.

133 All microcolonies were provided with an ad-lib supply of untreated pollen (Biobest via Agralan

134 Ltd, sterilised by gamma irradiation with a cobalt-60 source at dose rates between $25-45 \mathrm{kGy}$ ) 135 renewed every third day.

136 Three days after establishment of the microcolonies, 8 bees within each microcolony

137 individually received either a meal of $4 \mu 1$ of $30 \%$ sugar water (control and pesticide treatments)

138 or $30 \%$ sugar water containing a controlled dose of circa 130,000 N. ceranae spores (parasite 139 and pesticide+parasite treatments) (viability $98.6 \%$, viability test with $0.4 \%$ Trypan blue.

140 Viability of the spores was confirmed by infection of honeybees). Any remaining bees in the

141 microcolony were discarded. The rationale for only treating 8 bees was that we expected a small 142 percentage of workers to die prior to inoculation, so each microcolony contained 2 surplus bees 143 to ensure that an equal number per microcolony would be available to be treated. The dose 144 administered is typical of that used in honeybee studies (e.g. Alaux et al., 2010; Doublet et al., 145 2015) and dosages less than 100,000 spores have been found to infect bumblebees (Graystock et 146 al., 2013). Bees were starved for 2 hours before treatment. To facilitate the delivery of the meal, 147 bees were first immobilized by placing them in a cooler bag with ice blocks for approximately 148 10-15 minutes. Recovering bees ingested the inoculum when their proboscis was touched with a 149 droplet of the spore solution at the tip of a micropipette. A solution (in $\mathrm{dd}_{2} \mathrm{O}$ ) of freshly isolated 150 N. ceranae spores (originating from a naturally infected honeybee hive from Spain) was obtained 151 by homogenising abdomens of adult honeybees from a naturally infected colony, and purifying 152 the homogenate by centrifugation in 95\% Percoll® (Sigma-Aldrich). Identity of the parasite was 153 confirmed by PCR. After parasite treatment, microcolonies were monitored for worker mortality 154 and production of males every third day for 3 weeks, and then daily for 10 days. Newly emerged 155 males were removed from the microcolonies. The number of eggs, larvae and pupae were 
156 counted at the end of the experiment. Fecundity of workers was measured as the total number of

157 males, eggs, larvae and pupae produced during the experimental period. After the experiment, a

158 subset of alive $(\mathrm{n}=177)$ and dead bees $(\mathrm{n}=57)$ were screened for $N$. ceranae infection (on

159 average 5 bees per microcolony) as described above.

160

161

PER assays and memory retention

162 Proboscis extension response (PER) assay is a standard assay of learning ability in bees, which

163 tests their ability to learn an association between an odour (conditioned stimulus, CS) and sugar

164 reward (unconditioned stimulus, US) (Bitterman et al., 1983; Laloi et al., 1999). PER learning

165 assays began 7 days after the parasite treatment (10 days after the start of chronic pesticide

166 exposure). The day before PER conditioning started, workers were placed in a cooler bag with

167 ice blocks for approximately 10-15 minutes until immobilized, before being harnessed in plastic

168 tubes modified from $1 \mathrm{ml}$ pipette tips. The head of the bee was held in place using a "yoke"

169 made from a paper clip (see Riveros and Gronenberg, 2009). Harnessed bees were fed to

170 satiation with $60 \%$ sugar solution and then starved for 15 hours. In total 3 workers per

171 microcolony were harnessed. The following day bees were first allowed 20 minutes to acclimate

172 to the conditions of the room in which the PER assays were conducted (mean temp $23^{\circ} \mathrm{C}$ ). Then

173 one bee at a time was placed at $5 \mathrm{~cm}$ distance from a continuous air flow (circa $2 \mathrm{~L} \mathrm{~min}^{-1}$,

174 aquarium pump, Hidom, Shenzhen, China) delivered by a silicon tube (diameter $4 \mathrm{~mm}$ ) with a 1

$175 \mathrm{ml}$ pipette tip at the end. A floral odour (CS) linalool (Sigma-Aldrich) was then delivered by

176 switching the air flow pass through a $20 \mathrm{ml}$ syringe containing a $2 \times 20 \mathrm{~mm}$ filter paper with $5 \mu \mathrm{l}$

$1772 \mathrm{M}$ of the odorant in mineral oil. An extractor fan was placed behind the bee to allow extraction

178 of any residual odour. One PER assay was composed of $10 \mathrm{CS}-\mathrm{US}$ trials with each trial

179 conducted as follows: $15 \mathrm{~s}$ air flow, $3 \mathrm{~s}$ CS (air flow with odour only), $3 \mathrm{~s}$ both CS and US

180 (unconditioned stimulus: touching the antennae and proboscis with a toothpick dipped in $60 \%$

181 sugar solution (not spiked with pesticide) and allowing the bee to lick), $1 \mathrm{~s}$ US only, $8 \mathrm{~s}$ air flow.

182 The inter-trial interval (ITI) was 10 minutes. Extension of the proboscis during each CS and US

183 was recorded. After 10 CS-US trials, a final level of learning acquisition was assessed by

184 recording whether a bee extended its proboscis when presented with only the conditioned

185 stimulus without the sugar reward (US). 
To test whether bees remembered the learned association, a memory retention test was conducted 2 hours after each PER assay, where again only the conditioned stimulus was presented, in the absence of the US. 10 minutes before and after each PER assay and after each memory retention test, bees were tested for motivation to respond to sugar stimulus by touching the antennae with a toothpick covered with $60 \%$ sugar solution and observing whether extension of proboscis occurred. Bees that showed a negative response to the sugar solution were excluded

192 from the analyses. Also, bees that showed 4 or more sequential negative responses to US during PER assays were considered unmotivated and were excluded from analyses. After testing memory retention, bees were released from the harness, returned to their microcolony and their survival was observed.

\section{Statistics}

198 We used generalized (GLMM) and linear (LMM) mixed effect models in IBM SPSS v 21 (IBM SPSS Inc., USA) to analyse the effect of pesticide exposure and parasite treatment on measures of learning, fecundity and sugar water collection. For models with repeated measures structure (learning performance, sugar water collection), bee or microcolony identity was added as subject and trial number (trials 2-10) or the time point of sugar water measurement $(6,9,12,15,18,21$ and 24 days) as a repeated variable. In the analysis of learning performance, the first trial of the 10 CS-US trials was omitted to increase model fit (because it contained only zeroes). Binomial error structure with a logit link function was used in models analysing learning performance, final acquisition of learning (11th trial) and memory retention. A negative binomial error distribution with a log link was used to account for overdispersion in the models when the response variable was number of positive responses to CS and fecundity. In the analysis of sugar water collection, assumptions of homogeneity and normality of residuals were checked by inspecting residual plots (residuals against predicted values) and qq-plots. Bumblebee queenright nest (the original nest from which workers were divided into microcolonies) was considered as a random variable in the models. We first fitted the full model after which interaction terms were

213 omitted if they did not increase the model fit based on Akaike information criterion (AIC). AIC

214 was also used in selecting the repeated covariance type in models with repeated measures

215 structure. Significant interactions were post hoc tested with simple effects tests. 
216 Worker survival (dead, alive at the end of the experiment) was analysed with a generalized

217 linear mixed effects model (GLMM) with Laplace estimation and a logit link function in R

218 (version 3.1.3) (R Development Core Team, 2015) using the glmer function from the package

219 lme4 (Bates et al., 2014). Pesticide exposure and parasite treatment as well as harnessing

220 (harnessed for PER or not) were entered as fixed variables. Bumblebee queenright nest and

221 microcolony were included as random effects. We first fitted the full model, interaction terms

222 were then removed if they did not significantly increase the model fit based on maximum

223 likelihood ratio test. Significant interactions were further examined in post hoc tests performed

224 within each factor.

225

226

\section{Results}

227

228

\section{Nosema inoculations}

229 PCR screenings of a subset of alive and dead bees $(38.3 \%, 36.6 \%, 55.8 \%$, and $64.1 \%$ of the bees

230 in the control, pesticide, parasite and pesticide+parasite groups, respectively) showed that only 1

231 bee belonging to the parasite treatment group was infected with $N$. ceranae. No spores were

232 detected under the microscope. Due to potential stressful effects ingested parasite spores may

233 exert on bees, even though the bees were able to resist the infection (see discussion), this parasite

234 treatment is treated as a separate group in the results reported below.

\section{PER and memory retention}

237 Fifty four percent (97 out of 180) of harnessed bees successfully completed PER training. 19.4\%

238 of bees died during the starvation period and the remainder $26.7 \%$ were not sufficiently

239 responsive to the US stimulus. Learning performance, measured as the proportion of positive

240 proboscis extension responses to the odorant, conditioned stimulus (CS), at each trial, increased

241 during the $10 \mathrm{CS}-\mathrm{US}$ trials reaching $59.7 \%$ overall at the $10^{\text {th }}$ conditioning trial (Tables 1,2 , Fig.

242 1). Pesticide exposure or parasite treatment (note, bees did not become infected) did not affect

243 learning performance (Table 1). The motivation of bees to respond to the US stimulus remained

244 high throughout the $10 \mathrm{CS}-\mathrm{US}$ trials increasing overall from $82.5 \%$ to $96.9 \%$ (GLMM, F =

24535.32 , d.f. $=1,966, \mathrm{P}<0.001)$. There was no difference among treatment groups in the 
246 motivation to respond to the US (GLMM, Pesticide: $\mathrm{F}=3.012$, d.f. $=1,966, \mathrm{P}=0.09$; Parasite:

$247 \mathrm{~F}=1.28$, d.f. $=1,966, \mathrm{P}=0.26)$.

248 Overall, $68 \%$ of bees showed a positive proboscis extension response in the final test, 249 where bees were presented with the CS-only (trial 11, final acquisition level, Table 2, Fig. 1).

250 There was no difference among treatment groups in the final level of acquisition (GLMM, 251 Pesticide: $\mathrm{F}=0.002$, d.f. $=1,94, \mathrm{P}=0.97$; Parasite: $\mathrm{F}=0.01$, d.f. $=1,94, \mathrm{P}=0.91$ ) or in the 252 total number of positive PERs to CS (GLMM, Pesticide: $\mathrm{F}=0.11$, d.f. $=1,94, \mathrm{P}=0.74$; Parasite: $253 \mathrm{~F}=0.28$, d.f. $=1,94, \mathrm{P}=0.60)$.

254 Overall, $34 \%(n=85)$ of the tested bees remembered the association between the odour 255 and sugar reward when tested 2 hours after PER conditioning (Table 2, Fig. 1). There was no 256 difference in memory retention among treatment groups (GLMM, Pesticide: $\mathrm{F}=0.002$, d.f. $=1$, 257 82, $\mathrm{P}=0.96$; Parasite: $\mathrm{F}=0.68$, d.f. $=1,82, \mathrm{P}=0.41)$.

258

259

\section{Sugar water collection}

260 There was a significant interaction between parasite treatment and time point on sugar water collection by bees (LMM, F $=4.75$, d.f. $=6,58, P=0.001$, repeated covariance type: unstructured) indicating that the influence of ingestion of $N$. ceranae spores on sugar water collection was time-dependent (Fig. 2). While sugar water collection increased with time, it did not differ between parasite treated and control microcolonies at the beginning of the experiment (at 6 days: $\mathrm{P}=0.21$ ). However, parasite treated microcolonies collected less sugar water than control ones at measurement days 9 and 12 (both, $\mathrm{P}=0.003$ ) after which no differences were observed (all time points, $\mathrm{P}>0.32$, Fig. 2). Pesticide exposure did not affect sugar water collection $(\mathrm{F}=0.23$, d.f. $=1,41.4, \mathrm{P}=0.64)$.

\section{Fecundity}

271 Neither pesticide exposure nor parasite treatment affected fecundity (total number of males, 272 eggs, larvae and pupae produced by workers in microcolonies) (GLMM, Pesticide: F = 1.24, d.f. $273=1,57, \mathrm{P}=0.27$ : Parasite: $\mathrm{F}=0.55$, d.f. $=1,57, \mathrm{P}=0.46)$. Fecundity was on average $99.27 \pm$ 27413.44 (1 s.e.m.), $111.67 \pm 14.90,89.07 \pm 10.80$ and 101.47 \pm 12.44 for control, pesticide, 275 parasite and pesticide+parasite groups, respectively. 


\section{Survival}

278 There was a significant interaction between pesticide exposure and harnessing (Table 3)

279 indicating that the influence of pesticide exposure on survival depended on whether bees were

280 harnessed or not for PER assays (Fig. 3). Post hoc analysis revealed that pesticide exposure

281 decreased survival of bees that were harnessed but not in bees that were not harnessed

282 (Harnessed: $\mathrm{z}=-2.00, \mathrm{P}=0.04$ : Not harnessed; $\mathrm{z}=1.33, \mathrm{P}=0.18$ ). In general, bees that were not

283 harnessed for PER assay had much higher survival than those harnessed for PER. Parasite

284 treatment did not affect survival of bees $(\mathrm{z}=-0.23, \mathrm{P}=0.82)$.

285

\section{Discussion}

287 Given the importance of pollinators on ecosystem services, there is an urgent need to understand 288 the causes of pollinator declines (González-Varo et al., 2013; Goulson et al., 2015). We studied

289 the effect of chronic exposure to field-realistic sub-lethal levels of clothianidin on bumblebee

290 learning, fecundity and survival. Exposure to $1 \mathrm{ppb}$ clothianidin in sugar water had no significant

291 effects on the traits studied other than an increase in mortality when combined with the stress of

292 harnessing for learning assays.

293 Our results are in line with some previous studies that have observed no adverse effects of

294 sub-lethal, field-realistic exposure of neonicotinoids on bumblebee mortality or reproduction.

295 Exposure of $B$. impatiens colonies to 6 or $36 \mathrm{ppb}$ of clothianidin (8 weeks in pollen/sugar water

296 mixture) did not cause any significant reduction in the production of brood, workers, males or

297 queens (Franklin, Winston \& Morandin, 2004). Also, sub-lethal exposure to another

298 neonicotinoid compound, thiamethoxam, did not cause adverse effects on the reproduction or

299 mortality at exposure levels below 10 ppb (28 days in pollen and sugar water: Elston, Thompson

$300 \&$ Walters, 2013, 17 days in sugar water: Laycock et al., 2014) in contrast to imidacloprid (12

301 weeks in pollen and sugar water: Tasei, Lerin \& Ripault, 2000, 13-14 days in sugar water:

302 Laycock et al., 2012; Laycock \& Cresswell, 2013), to which bumblebees may be more sensitive

303 (Laycock et al., 2014). However, there is contrasting evidence as to whether sub-lethal exposure

304 to thiamethoxam or to its major metabolite, clothianidin, has harmful effects on bumblebees.

305 Fauser-Misslin et al. (2014) found that exposure for 9 weeks to $1.5 \mathrm{ppb}$ clothianidin and $4 \mathrm{ppb}$

306 thiamethoxam reduced worker production and longevity of bumblebee colonies in the laboratory.

307 Furthermore, a recent field experiment by Rundlöf et al. (2015) revealed that exposure to 
308 clothianidin in seed coated oilseed rape was associated with lower wild bumblebee densities and

309 negatively affected colony growth and reproduction in commercially reared bumblebee nests

310 (monitored for 40 days). The mean concentration of clothianidin in bee-collected nectar was 5.4

311 ppb (range 1.4 -14 ppb) (Rundlöf et al., 2015), which is higher than that used in our study (1

$312 \mathrm{ppb}$ ) and higher than residue levels typically found in nectar of treated crops (Godfray et al.,

313 2014; Sanchez-Bayo \& Goka, 2014; Bonmatin et al., 2015). Overall, it appears that exposure to 1

314 ppb clothianidin used in our study is not harmful to bumblebees in a laboratory setting, whereas

315 slightly higher exposure levels combined with other environmental stressors can result in

316 impaired fitness effects (Fauser-Misslin et al., 2014; Rundlöf et al., 2015). It is also worth noting

317 that we only spiked sugar water and used only one neonicotinoid whereas Fauser-Misslin et al.,

318 (2014) also spiked pollen and exposed bees to two different neonicotinoids.

319 Even though reproduction was not affected, sub-lethal effects of pesticide exposure may

320 be manifested in other traits such as behaviour, where subtle changes in behaviour or learning

321 may result in significant impacts at the colony level (Raine \& Chittka, 2008). However, our PER

322 assays did not reveal any significant effects on learning or memory at the dose used. Recently, a

323 laboratory study exposing bumblebees to as low as $2.5 \mathrm{ppb}$ thiamethoxam for 3.5 weeks was

324 found to impair olfactory learning (Stanley, Smith \& Raine, 2015). In honeybees, both negative

325 and positive effects of pesticides on olfactory learning ability have been reported (Decourtye et

326 al., 2004; Williamson \& Wright, 2013; Williamson, Baker \& Wright, 2013). For instance, four

327 days chronic exposure to sub-lethal concentrations of imidacloprid (10 $\left.\mathrm{nmol} \mathrm{1}^{-1} \sim 2.3 \mathrm{ppb}\right)$

328 reduced learning acquisition in A. mellifera (Williamson \& Wright, 2013) and a single acute dose

329 of $0.1 \mathrm{ng}$ imidacloprid was sufficient to impair learning acquisition in the Asian honeybee $A$.

330 cerana (Tan et al., 2015). Interestingly, in our study, pesticide exposed bumblebees had slightly,

331 but not significantly, faster acquisition rate compared to the other treatment groups, suggesting

332 possible hormetic effects (Cutler \& Rix, 2015). It would be useful to follow up our study by

333 studying learning of bumblebees when exposed to a range of concentrations of pesticide,

334 although it should be noted that PER assays are very labour intensive to perform.

335 Interestingly, pesticide exposure in combination with additional stress caused by

336 harnessing bees for 15 hours and conducting learning assays increased mortality. Overall, PER

337 tested bees had higher mortality compared to those not harnessed and tested in the PER assay. It

338 is not clear whether cold stress, harnessing, starvation prior to the assay, some facet of the PER 
339 test itself, or possibly a combination of these factors results in a higher risk of mortality. It is

340 known that cold exposure can be stressful for the bees (Poissonnier, Jackson \& Tanner, 2015).

341 However, only $19.4 \%$ of the bees died during the 15 hour starvation period (after cold narcosis),

342 the majority of bees $(37.2 \%)$ dying within the 3 days following PER testing. This indicates that

343 cold exposure and/or starvation stress did not cause high immediate mortality, but nonetheless

344 may have inflicted measurable harm leading to delayed mortality, particularly in bees also

345 exposed to pesticides. The possible interactive effects of pesticide with additional stress observed

346 here would demand further investigation.

347 Rather unexpectedly, the bumblebees in our study did not become infected by $N$. ceranae.

348 There are only few studies that have experimentally investigated virulence of $N$. ceranae in

349 bumblebees with infection levels shown to vary substantially, from about 30\% (Fürst et al.,

3502014 ) to over 50\% (Graystock et al., 2013), with survival ranging from $100 \%$ to $35 \%$,

351 respectively. It could be that the $N$. ceranae strains used in our study were either not infective

352 towards bumblebees, or that the commercial bumblebee strains used were resistant to this gut

353 parasite. Both the host and the parasite play an essential role in determining the outcome of an

354 infection (Frank \& Schmid-Hempel, 2008) in which the nature of fitness trade-offs between them

355 may determine the level of virulence. At the same time, Nosema strains vary in infectivity and

356 virulence (Genersch, 2010) and several studies have reported different degrees of tolerance and

357 resistance to $N$. ceranae associated with the genetic background of the honeybee host (Dussaubat

358 et al., 2013; Fontbonne et al., 2013; Huang et al., 2013, 2014). It should also be noted that the

359 Nosema strain we used in our study was able to infect honeybees inoculated with the same dose

360 as used in the experiment (results not shown), indicating that the very low infectivity in

361 bumblebees is not because the spores were inviable.

362 Even though bees were not infected by N. ceranae, the parasite inoculation had slight,

363 short-term effect on sugar water collection. Reasons for reduced collection of sugar water 6-9

364 days after Nosema treatment (9-12 days after starting pesticide feeding) are not clear. In

365 honeybees, Nosema challenge has been linked to increased energetic stress leading to increased

366 feeding rates (Naug \& Gibbs, 2009; Mayack \& Naug, 2009; Martín-Hernández et al., 2011).

367 However, as the inoculation of the bumblebees with viable $N$. ceranae spores did not lead to

368 infection in our experiment, the same outcome cannot be expected. Interestingly, previous

369 studies have observed stimulation of the bumblebee immune system just a few hours after 
370 ingesting food containing pathogenic microorganisms (Riddell et al., 2011; Brunner, Schmid-

371 Hempel \& Barribeau, 2013), and even when fed non-pathogenic elicitors such as

372 lipopolysaccharides (Moret \& Schmid-Hempel, 2000). Therefore, we postulate that the short-

373 term reduction in the collection of sugar water in microcolonies treated with Nosema may be due

374 to some behavioural or physiological alterations related to the stimulation of the immune

375 response in the host after contact with the parasite (Alghamdi et al., 2008), though further

376 research would be needed to confirm this. Overall, our results on Nosema emphasizes the need

377 for further research on infectivity and virulence of $N$. ceranae in bumblebees in order to assess

378 whether $N$. ceranae presents a serious threat to bumblebee health.

379

380

\section{Acknowledgements}

381 We thank Dezene Huber and three anonymous reviewers for their constructive and helpful

382 comments, and are grateful to Mariano Higes, Raquel Martín-Hernández, Cristina Rodríguez-

383 García and Teresa Corrales from CIAPA Research Institute for providing Nosema spores.

384

385

\section{References}

386

387

388

389

390

391

392

393

394

395

396

397

398

399

400

Alaux C, Brunet J-L, Dussaubat C, Mondet F, Tchamitchan S, Cousin M, Brillard J, Baldy A, Belzunces LP, Le Conte Y. 2010. Interactions between Nosema microspores and a neonicotinoid weaken honeybees (Apis mellifera). Environmental Microbiology 12:774782.

Alghamdi A, Dalton L, Phillis A, Rosato E, Mallon EB. 2008. Immune response impairs learning in free-flying bumble-bees. Biology Letters 4:479-481.

Aliouane Y, El Hassani A, Gary V, Armengaud C, Lambin M, Gauthier M. 2009. Subchronic exposure of honeybees to sublethal doses of pesticides: effects on behaviour. Environmental Toxicology and Chemistry 28:113-122.

Bates DM, Mächler M, Bolker B, Walker SC. 2014. Ime4: Linear mixed-effects models using Eigen and S4. Package version 1.1-7.

Biesmeijer JC, Roberts SPM, Reemer M, Ohlemüller R, Edwards M, Peeters T, Schaffers a P, Potts SG, Kleukers R, Thomas CD, Settele J, Kunin WE. 2006. Parallel declines in pollinators and insect-pollinated plants in Britain and the Netherlands. Science 313:351354. 
401

402

403

404

405

406

407

408

409

410

411

412

413

414

415

416

417

418

419

420

421

422

423

424

425

426

427

428

429

430

431

432

433

434

Bitterman ME, Menzel R, Fietz A, Schäfer S. 1983. Classical conditioning of proboscis extension in honeybees (Apis mellifera). Journal of Comparative Psychology 97:107-119.

Bonmatin J-M, Giorio C, Girolami V, Goulson D, Kreutzweiser DP, Krupke C, Liess M, Long E, Marzaro M, Mitchell EAD, Noome DA, Simon-Delso N, Tapparo A. 2015. Environmental fate and exposure; neonicotinoids and fipronil. Environmental Science and Pollution Research 22:35-67.

Botías C, David A, Horwood J, Abdul-Sada A, Nicholls E, Hill EM, Goulson D. 2015. Neonicotinoid residues in wildflowers, a potential route of chronic exposure for bees. Environmental Science \& Technology 49:12731-12740.

Brunner FS, Schmid-Hempel P, Barribeau SM. 2013. Immune gene expression in Bombus terrestris: signatures of infection despite strong variation among populations, colonies, and sister workers. PLoS One 8:e68181.

Cresswell JE. 2011. A meta-analysis of experiments testing the effects of a neonicotinoid insecticide (imidacloprid) on honey bees. Ecotoxicology 20:149-157.

Cresswell JE, Page CJ, Uygun MB, Holmbergh M, Li Y, Wheeler JG, Laycock I, Pook CJ, de Ibarra NH, Smirnoff N, Tyler CR. 2012. Differential sensitivity of honey bees and bumble bees to a dietary insecticide (imidacloprid). Zoology 115:365-371.

Cutler GC, Scott-Dupree CD, Sultan M, McFarlane AD, Brewer L. 2014. A large-scale field study examining effects of exposure to clothianidin seed-treated canola on honey bee colony health, development, and overwintering success. PeerJ 2:e652.

Cutler GC, Rix RR. 2015. Can poisons stimulate bees? Appreciating the potential of hormesis in bee-pesticide research. Pest Management Science 71:1368-1370.

Decourtye A, Armengaud C, Renou M, Devillers J, Cluzeau S, Gauthier M, Pham-Delègue M-H. 2004. Imidacloprid impairs memory and brain metabolism in the honeybee (Apis mellifera L.). Pesticide Biochemistry and Physiology 78:83-92.

Doublet V, Labarussias M, de Miranda JR, Moritz RF A., Paxton RJ. 2015. Bees under stress: sublethal doses of a neonicotinoid pesticide and pathogens interact to elevate honey bee mortality across the life cycle. Environmental Microbiology 17:969-983.

Dussaubat C, Sagastume S, Gómez-Moracho T, Botías C, García-Palencia P, Martín-Hernández R, Le Conte Y, Higes M. 2013. Comparative study of Nosema ceranae (Microsporidia) isolates from two different geographic origins. Veterinary Microbiology 162:670-678.

Elston C, Thompson HM, Walters KFA. 2013. Sub-lethal effects of thiamethoxam, a neonicotinoid pesticide, and propiconazole, a DMI fungicide, on colony initiation in bumblebee (Bombus terrestris) micro-colonies. Apidologie 44:563-574. 
435

436

437

438

439

440

441

442

443

444

445

446

447

448

449

450

451

452

453

454

455

456

457

458

459

460

461

462

463

464

465

466

467

European Food Safety Authority. 2012. Statement on the findings in recent studies investigating sub-lethal effects in bees of some neonicotinoids in consideration of the uses currently authorised in Europe. EFSA Journal 10:2752.

European Food Safety Authority. 2013. EFSA guidance document on the risk assessment of plant protection products on bees (Apis mellifera, Bombus spp. and solitary bees). EFSA Journal 11:3295.

Fauser-Misslin A, Sadd BM, Neumann P, Sandrock C. 2014. Influence of combined pesticide and parasite exposure on bumblebee colony traits in the laboratory. Journal of Applied Ecology 51:450-459.

Feltham H, Park K, Goulson D. 2014. Field realistic doses of pesticide imidacloprid reduce bumblebee pollen foraging efficiency. Ecotoxicology 23:317-323.

Fischer J, Müller T, Spatz A-K, Greggers U, Grünewald B, Menzel R. 2014. Neonicotinoids interfere with specific components of navigation in honeybees. PLoS One 9:e91364.

Fontbonne R, Garnery L, Vidau C, Aufauvre J, Texier C, Tchamitchian S, El Alaoui H, Brunet JL, Delbac F, Biron DG. 2013. Comparative susceptibility of three Western honeybee taxa to the microsporidian parasite Nosema ceranae. Infection, Genetics and Evolution 17:188194.

Frank SA, Schmid-Hempel P. 2008. Mechanisms of pathogenesis and the evolution of parasite virulence. Journal of Evolutionary Biology 21:396-404.

Franklin MT, Winston ML, Morandin LA. 2004. Effects of clothianidin on Bombus impatiens (Hymenoptera: Apidae) colony health and foraging ability. Journal of Economic Entomology 97:369-373.

Fürst MA, McMahon DP, Osborne JL, Paxton RJ, Brown MJF. 2014. Disease associations between honeybees and bumblebees as a threat to wild pollinators. Nature 506:364-366.

Gallai N, Salles J-M, Settele J, Vaissière BE. 2009. Economic valuation of the vulnerability of world agriculture confronted with pollinator decline. Ecological Economics 68:810-821.

Gegear RJ, Otterstatter MC, Thomson JD. 2006. Bumble-bee foragers infected by a gut parasite have an impaired ability to utilize floral information. Proceedings of the Royal Society BBiological Sciences 273:1073-1078.

Genersch E. 2010. Honey bee pathology: current threats to honey bees and beekeeping. Applied Microbiology and Biotechnology 87:87-97.

Gill RJ, Raine NE. 2014. Chronic impairment of bumblebee natural foraging behaviour induced by sublethal pesticide exposure. Functional Ecology 28:1459-1471. 
468

Gill RJ, Ramos-Rodriguez O, Raine NE. 2012. Combined pesticide exposure severely affects individual- and colony-level traits in bees. Nature 491:105-108.

Godfray HCJ, Blacquiere T, Field LM, Hails RS, Petrokofsky G, Potts SG, Raine NE, Vanbergen AJ, McLean AR. 2014. A restatement of the natural science evidence base concerning neonicotinoid insecticides and insect pollinators. Proceedings of the Royal Society B-Biological Sciences 281:20140558.

Gómez-Moracho T, Bartolomé C, Bello X, Martín-Hernández R, Higes M, Maside X. 2015. Recent worldwide expansion of Nosema ceranae (Microsporidia) in Apis mellifera populations inferred from multilocus patterns of genetic variation. Infection, Genetics and Evolution 31:87-94.

González-Varo JP, Biesmeijer JC, Bommarco R, Potts SG, Schweiger O, Smith HG, SteffanDewenter I, Szentgyörgyi H, Woyciechowski M, Vilà M. 2013. Combined effects of global change pressures on animal-mediated pollination. Trends in Ecology \& Evolution 28:524530 .

Goulson D. 2010. Bumblebees; their behaviour, ecology and conservation. Oxford: Oxford University Press.

Goulson D. 2013. An overview of the environmental risks posed by neonicotinoid insecticides. Journal of Applied Ecology 50:977-987.

Goulson D, Nicholls E, Botías C, Rotheray EL. 2015. Bee declines driven by combined stress from parasites, pesticides, and lack of flowers. Science 347:1255957.

Graystock P, Yates K, Darvill B, Goulson D, Hughes WOH. 2013. Emerging dangers: deadly effects of an emergent parasite in a new pollinator host. Journal of Invertebrate Pathology 114:114-119.

Graystock P, Goulson D, Hughes WOH. 2014. The relationship between managed bees and the prevalence of parasites in bumblebees. PeerJ 2:e522.

Henry M, Béguin M, Requier F, Rollin O, Odoux J-F, Aupinel P, Aptel J, Tchamitchian S, Decourtye A. 2012. A common pesticide decreases foraging success and survival in honey bees. Science 336:348-350.

Higes M, García-Palencia P, Martín-Hernández R, Meana A. 2007. Experimental infection of Apis mellifera honeybees with Nosema ceranae (Microsporidia). Journal of Invertebrate Pathology 94:211-217.

Huang Q, Kryger P, Le Conte Y, Lattorff HMG, Kraus FB, Moritz RFA. 2013. Four quantitative trait loci associated with low Nosema ceranae (Microsporidia) spore load in the honeybee Apis mellifera. Apidologie 45:248-256. 
502 Huang Q, Lattorff HMG, Kryger P, Le Conte Y, Moritz RFA. 2014. A selective sweep in a

503

504

505

506

507

508

509

510

511

512

513

514

515

516

517

518

519

520

521

522

523

524

525

526

527

528

529

530

531

532

533

534

535 microsporidian parasite Nosema-tolerant honeybee population, Apis mellifera. Animal Genetics 45:267-273.

Jeschke P, Nauen R, Schindler M, Elbert A. 2011. Overview of the status and global strategy for neonicotinoids. Journal of Agricultural and Food Chemistry 59:2897-2908.

Kralj J, Brockmann A, Fuchs S, Tautz J. 2007. The parasitic mite Varroa destructor affects nonassociative learning in honey bee foragers, Apis mellifera L. Journal of Comparative Physiology A 193:363-370.

Krupke CH, Hunt GJ, Eitzer BD, Andino G, Given K. 2012. Multiple routes of pesticide exposure for honey bees living near agricultural fields. PLoS One 7:e29268.

Laloi D, Sandoz JC, Picard-Nizou AL, Marchesi A, Pouvreau A, Taséi JN, Poppy G, PhamDelègue MH. 1999. Olfactory conditioning of the proboscis extension in bumble bees. Entomologia Experimentalis et Applicata 90:123-129.

Laycock I, Lenthall KM, Barratt AT, Cresswell JE. 2012. Effects of imidacloprid, a neonicotinoid pesticide, on reproduction in worker bumble bees (Bombus terrestris). Ecotoxicology 21:1937-1945.

Laycock I, Cotterell KC, O'Shea-Wheller TA, Cresswell JE. 2014. Effects of the neonicotinoid pesticide thiamethoxam at field-realistic levels on microcolonies of Bombus terrestris worker bumble bees. Ecotoxicology and Environmental Safety 100:153-158.

Laycock I, Cresswell JE. 2013. Repression and recuperation of brood production in Bombus terrestris bumble bees exposed to a pulse of the neonicotinoid pesticide imidacloprid. PLoS One 8:e79872.

Martín-Hernández R, Botías C, Barrios L, Martínez-Salvador A, Meana A, Mayack C, Higes M. 2011. Comparison of the energetic stress associated with experimental Nosema ceranae and Nosema apis infection of honeybees (Apis mellifera). Parasitology Research 109:605-612.

Mayack C, Naug D. 2009. Energetic stress in the honeybee Apis mellifera from Nosema ceranae infection. Journal of Invertebrate Pathology 100:185-188.

Meeus I, Brown MJF, De Graaf DC, Smagghe G. 2011. Effects of invasive parasites on bumble bee declines. Conservation Biology 25:662-671.

Moffat C, Pacheco JG, Sharp S, Samson AJ, Bollan KA, Huang J, Buckland ST, Connolly CN. 2015. Chronic exposure to neonicotinoids increases neuronal vulnerability to mitochondrial dysfunction in the bumblebee (Bombus terrestris). FASEB Journal 29:2112-2119.

Morandin LA, Winston ML. 2003. Effects of novel pesticides on bumble bee (Hymenoptera: Apidae) colony health and foraging ability. Environmental Entomology 32:555-563. 
536 Moret Y, Schmid-Hempel P. 2000. Survival for immunity: the price of immune system activation for bumblebee workers. Science 290:1166-1168.

Naug D, Gibbs A. 2009. Behavioral changes mediated by hunger in honeybees infected with Nosema ceranae. Apidologie 40:595-599.

Ollerton J, Winfree R, Tarrant S. 2011. How many flowering plants are pollinated by animals? Oikos 120:321-326.

542

543

544

545

546

547

548

549

550

551

552

553

554

555

556

557

558

559

560

561

562

563

564

565

566 567

Palmer MJ, Moffat C, Saranzewa N, Harvey J, Wright GA, Connolly CN. 2013. Cholinergic pesticides cause mushroom body neuronal inactivation in honeybees. Nature Communications 4:1634.

Pilling E, Campbell P, Coulson M, Ruddle N, Tornier I. 2013. A four-year field program investigating long-term effects of repeated exposure of honey bee colonies to flowering crops treated with thiamethoxam. PLoS One 8:e77193.

Plischuk S, Martín-Hernández R, Prieto L, Lucía M, Botías C, Meana A, Abrahamovich AH, Lange C, Higes M. 2009. South American native bumblebees (Hymenoptera: Apidae) infected by Nosema ceranae (Microsporidia), an emerging pathogen of honeybees (Apis mellifera). Environmental Microbiology Reports 1:131-135.

Poissonnier L-A, Jackson AL, Tanner CJ. 2015. Cold and CO2 narcosis have long-lasting and dissimilar effects on Bombus terrestris. Insectes Sociaux 62:291-298.

Potts SG, Biesmeijer JC, Kremen C, Neumann P, Schweiger O, Kunin WE. 2010. Global pollinator declines: trends, impacts and drivers. Trends in Ecology \& Evolution 25:345-353.

R Development Core Team. 2015. R: a language and environment for statistical computing. Vienna, Austria: R foundation for Statistical Computing.

Raine NE, Chittka L. 2008. The correlation of learning speed and natural foraging success in bumble-bees. Proceedings of the Royal Society B-Biological Sciences 275:803-808.

Ravoet J, Maharramov J, Meeus I, De Smet L, Wenseleers T, Smagghe G, de Graaf DC. 2013. Comprehensive bee pathogen screening in Belgium reveals Crithidia mellificae as a new contributory factor to winter mortality. PloS one 8:e72443.

Riddell CE, Sumner S, Adams S, Mallon EB. 2011. Pathways to immunity: temporal dynamics of the bumblebee (Bombus terrestris) immune response against a trypanosomal gut parasite. Insect Molecular Biology 20:529-540.

Riveros AJ, Gronenberg W. 2009. Olfactory learning and memory in the bumblebee Bombus occidentalis. Naturwissenschaften 96:851-856. 
568 Rundlöf M, Andersson GKS, Bommarco R, Fries I, Hederström V, Herbertsson L, Jonsson O,

569

570

571

572

573

574

575

576

577

578

579

580

581

582

583

584

585

586

587

588

589

590

591

592

593

594

595

596

597

598

599

Klatt BK, Pedersen TR, Yourstone J, Smith HG. 2015. Seed coating with a neonicotinoid insecticide negatively affects wild bees. Nature 521:77-80.

Sanchez-Bayo F, Goka K. 2014. Pesticide residues and bees - a risk assessment. PLoS One 9:e94482.

Schmuck R, Schöning R, Stork A, Schramel O. 2001. Risk posed to honeybees (Apis mellifera L, Hymenoptera) by an imidacloprid seed dressing of sunflowers. Pest Management Science $57: 225-238$.

Schneider CW, Tautz J, Grünewald B, Fuchs S. 2012. RFID tracking of sublethal effects of two neonicotinoid insecticides on the foraging behavior of Apis mellifera. PLoS One 7:e30023.

Scholer J, Krischik V. 2014. Chronic exposure of imidacloprid and clothianidin reduce queen survival, foraging, and nectar storing in colonies of Bombus impatiens. PLoS One 9:e91573.

Smith ML. 2012. The honey bee parasite Nosema ceranae: transmissible via food exchange? PLoS One 7:e43319.

Stanley D a., Smith KE, Raine NE. 2015. Bumblebee learning and memory is impaired by chronic exposure to a neonicotinoid pesticide. Scientific Reports 5:16508.

Tan K, Chen W, Dong S, Liu X, Wang Y, Nieh JC. 2015. A neonicotinoid impairs olfactory learning in Asian honey bees (Apis cerana) exposed as larvae or as adults. Scientific Reports 5:10989.

Tasei J-N, Lerin J, Ripault G. 2000. Sub-lethal effects of imidacloprid on bumblebees, Bombus terrestris (Hymenoptera: Apidae), during a laboratory feeding test. Pest Management Science 56:784-788.

Tasei JN, Ripault G, Rivault E. 2001. Hazards of imidacloprid seed coating to Bombus terrestris (Hymenoptera: Apidae) when applied to sunflower. Journal of Economic Entomology 94:623-627.

Whitehorn PR, O'Connor S, Wackers FL, Goulson D. 2012. Neonicotinoid pesticide reduces bumble bee colony growth and queen production. Science 336:351-352.

Williamson SM, Baker DD, Wright GA. 2013. Acute exposure to a sublethal dose of imidacloprid and coumaphos enhances olfactory learning and memory in the honeybee Apis mellifera. Invertebrate Neuroscience 13:63-70.

Williamson SM, Wright GA. 2013. Exposure to multiple cholinergic pesticides impairs olfactory learning and memory in honeybees. The Journal of experimental biology 216:1799-1807. 
600 Yang E-C, Chang H-C, Wu W-Y, Chen Y-W. 2012. Impaired olfactory associative behavior of 601 honeybee workers due to contamination of imidacloprid in the larval stage. PLoS One $602 \quad 7: e 49472$.

603 Zars T. 2000. Behavioral functions of the insect mushroom bodies. Current Opinion in $604 \quad$ Neurobiology 10:790-795.

605 
606 Tables

607 Table 1 Results of repeated generalized linear mixed effect model (GLMM) on the learning

608 performance of $B$. terrestris workers chronically exposed to pesticide clothianidin and ingested

609 parasite $N$. ceranae spores.

\begin{tabular}{lrcc}
\hline Effect & \multicolumn{1}{l}{ F } & d.f. $(\mathrm{n}, \mathrm{d})$ & $\mathrm{P}$ \\
\hline Corrected model & 59.41 & 3,869 & $<0.001$ \\
Pesticide & 0.46 & 1,869 & 0.50 \\
Parasite & 0.55 & 1,869 & 0.46 \\
Trial number & 178.14 & 1,869 & $<0.001$ \\
\hline
\end{tabular}

610 Restricted maximum-likelihood procedure was used in the estimation. Random factor nest, 611 estimate \pm residual: $0.28 \pm 0.21, \mathrm{z}=1.30, \mathrm{P}=0.19$. Akaike information criterion (AIC) $=$

612 3666.30, repeated covariance type: compound symmetry. Interaction between pesticide and 613 parasite was non-significant $(\mathrm{F}(1,867)=0.20, \mathrm{P}=0.66)$ and was removed during model 614 selection.

615 
616 Table 2 Percentage of $B$. terrestris workers showing positive proboscis extension response

617 (PER) and total sample sizes at the 10th CS-US trial, 11th CS trial (final level of learning

618 acquisition) and after 2 hours in the memory retention test for different treatment groups.

619 Pesticide: bees chronically exposed to pesticide clothianidin. Parasite: bees ingested parasite $N$.

620 ceranae spores. Both: bees chronically exposed to pesticide clohianidin and ingested parasite $N$.

621 ceranae spores.

\begin{tabular}{lccc}
\hline Treatment group & $\begin{array}{c}\text { 10th Trial } \\
\text { \% PER (n) }\end{array}$ & $\begin{array}{c}\text { Final level of learning acquisition } \\
\text { \% PER (n) }\end{array}$ & $\begin{array}{c}\text { Memory retention test } \\
\text { \% PER (n) }\end{array}$ \\
\hline Control & $54.5(22)$ & $68.2(22)$ & $23.8(21)$ \\
Pesticide & $69.6(23)$ & $69.6(23)$ & $36.8(19)$ \\
Parasite & $57.7(26)$ & $69.2(26)$ & $43.5(23)$ \\
Both & $57.7(26)$ & $65.4(26)$ & $31.8(22)$ \\
\hline
\end{tabular}

622

623 
624 Table 3 Results of a generalized linear mixed effect model (GLMM) on survival of B. terrestris

625 workers exposed to pesticide clothianidin and ingested parasite $N$. ceranae spores, and harnessed 626 for proboscis extension response (PER) assay.

\begin{tabular}{lcccc}
\hline Effect & Estimate & s.e.m & Z & P \\
\hline Pesticide & 0.76 & 0.48 & 1.61 & 0.11 \\
Parasite & -0.08 & 0.37 & -0.23 & 0.82 \\
Harnessing & -2.24 & 0.37 & -6.06 & $<0.001$ \\
Pesticide x Harnessing & -1.46 & 0.54 & -2.74 & 0.006 \\
\hline
\end{tabular}

627 


\section{Figures}

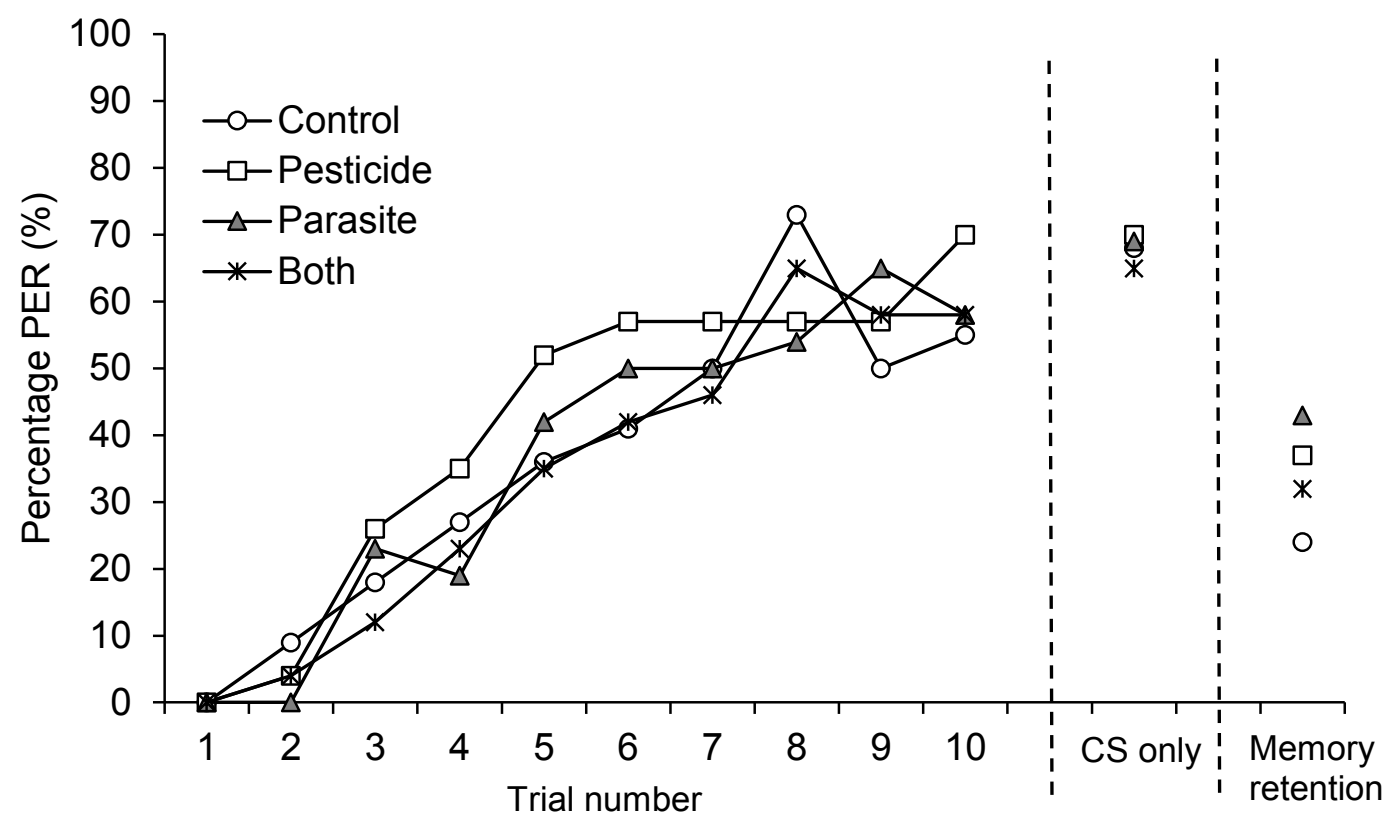

629

630 Fig. 1 Percentage (\%) of $B$. terrestris bumblebee workers showing proboscis extension response 631 (PER) to odour (CS, conditioned stimulus) stimulation across 10 CS-US (US: unconditioned

632 stimulus) trials for bees exposed to pesticide clothianidin and ingested parasite $N$. ceranae

633 spores. CS only: percentage of PER at the $11^{\text {th }}$ CS trial. Memory retention: percentage of PER 2 634 hours after learning acquisition

635 


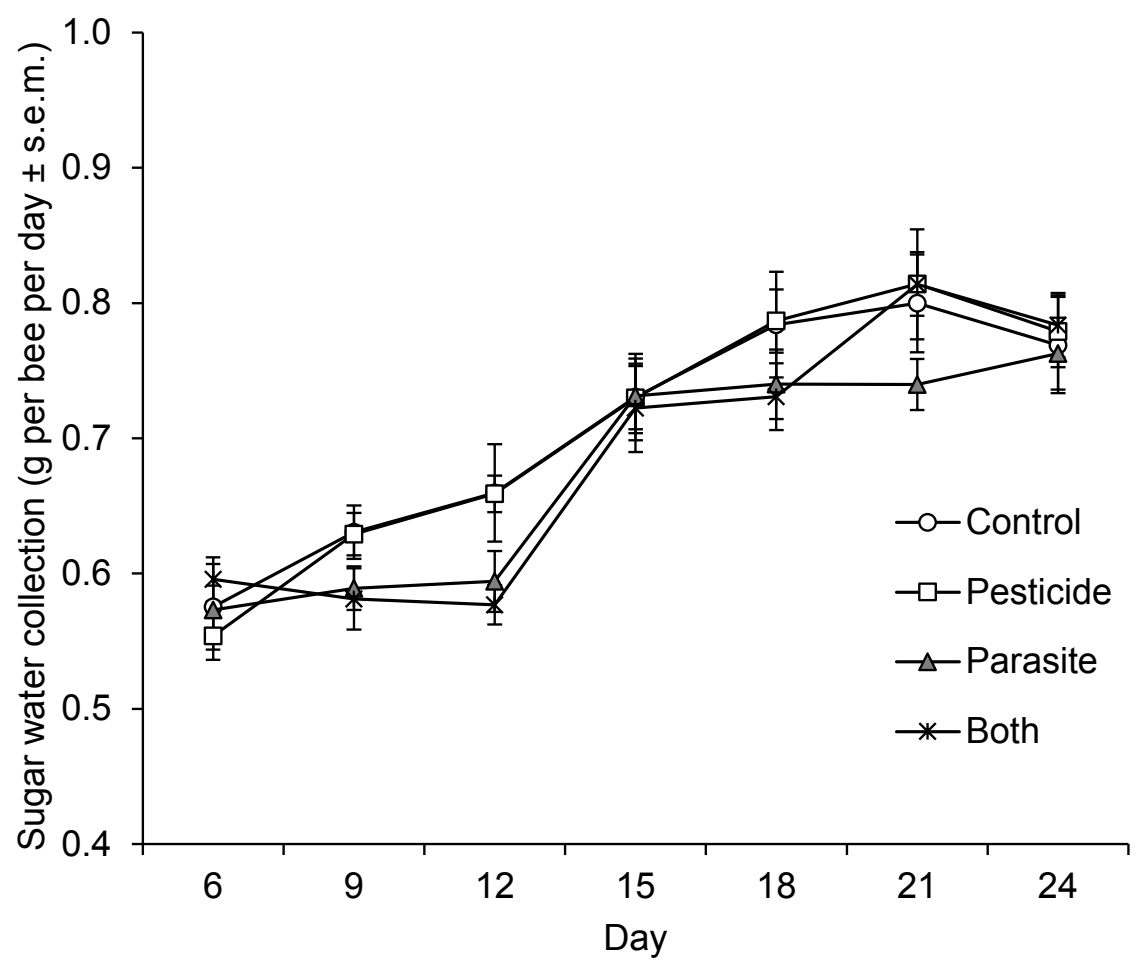

636

637 Fig. 2 Sugar water collection of $B$. terrestris bumblebee microcolonies exposed to pesticide 638 clothianidin and ingested parasite N. ceranae spores.

639 


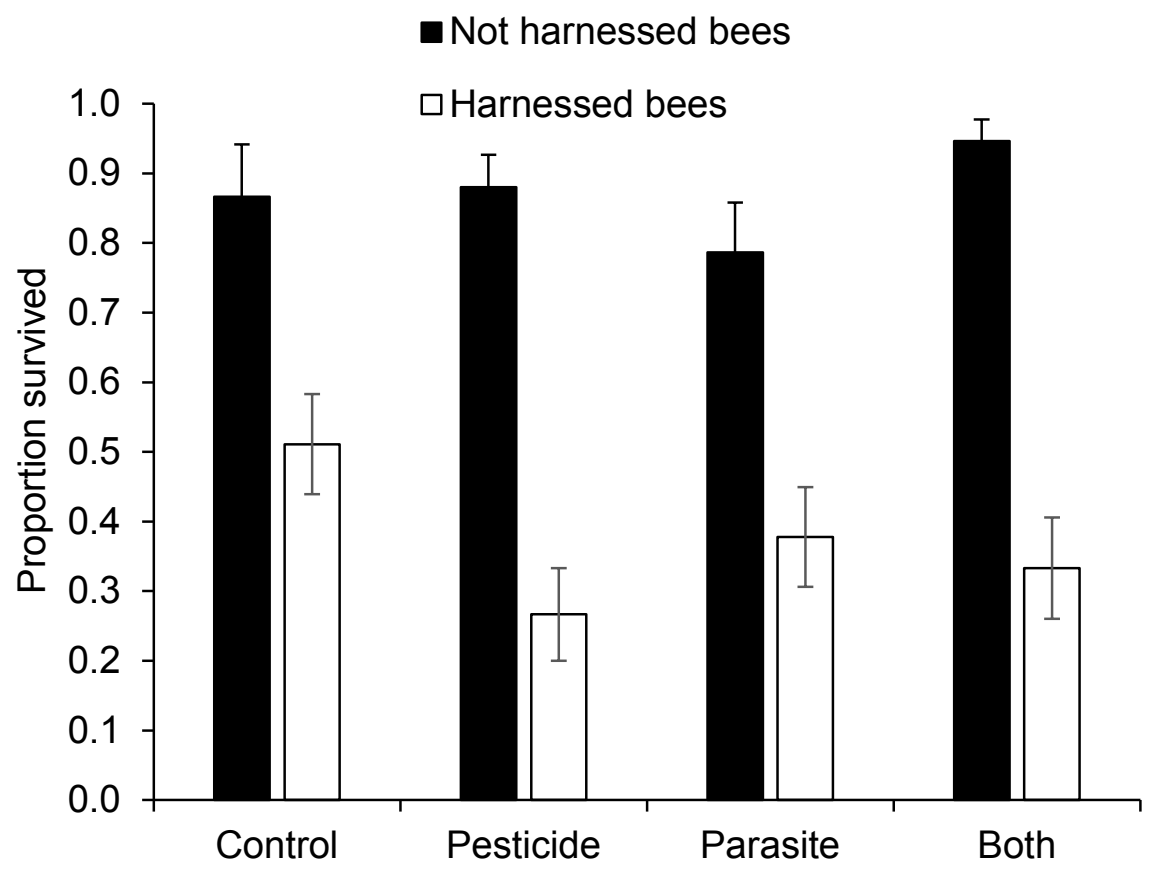

641 Fig. 3 Survival (mean \pm s.e.m) of $B$. terrestris bumblebee workers harnessed and not-harnessed

642 for proboscis extension response (PER) analysis, and exposed to pesticide clothianidin and 643 ingested parasite N. ceranae spores. 